\title{
End-of-Life Inventory Problem with Phase-out Returns
}

\author{
M. Pourakbar ${ }^{\dagger}$, E. van der Laan ${ }^{\ddagger}$, R. Dekker ${ }^{\dagger *}$ \\ $\dagger$ Erasmus School of Economics, $\ddagger$ Rotterdam School of Management \\ Erasmus University Rotterdam \\ $†$ Burgemeester Oudlaan 50, 3000DR Rotterdam, the Netherlands \\ pourakbar@ese.eur.nl, laan@rsm.nl,rdekker@ese.eur.nl \\ * Phone: (+31)10-4081274, Fax: (+31)10-4089162
}

\section{Econometric Institute Report EI 2011-12}

\begin{abstract}
We consider the service parts end-of-life inventory problem of a capital goods manufacturer in the final phase of its life cycle. The final phase starts as soon as the production of parts terminates and continues till the last service contract expires. Final order quantities are considered a popular tactic to sustain service fulfillment obligations and to mitigate the effect of obsolescence. In addition to the final order quantity, other sources to obtain serviceable parts are repairing returned defective items and retrieving parts from phase-out returns. Phase-out returns happen when a customer replaces an old system platform with a next generation one and returns the old product to the original equipment manufacturer (OEM). These returns can well serve the demand for service parts of other customers still using the old generation of the product. In this paper, we study the decision making complications stemming from phase-out occurrence. We use a finite horizon Markov decision process to characterize the structure of
\end{abstract}


the optimal inventory control policy. We show that the optimal policy consists of a time varying threshold level for item repair. Furthermore, we study the value of phase-out information by extending the results to cases with an uncertain phase-out quantity or an uncertain schedule. Numerical analysis sheds light on the advantages of the optimal policy compared to some heuristic policies.

Keywords: End-of-life inventory management, spare parts, phase-out returns

\section{Introduction}

Due to the spurt of technology and innovation the service life cycle of both parts and products have become shorter. Consequently, managing the inventories of service parts in order to fulfill service obligations and avoid obsolescence risk has become a major challenge for companies. This is even more crucial as the production of a service part is discontinued when the part enters its final phase of service life cycle. In this phase, various strategic decisions can be made to keep the product in the market. These include substituting another part for the obsolete one, obtaining the obsolete part from an after market manufacturer, redesigning the product, discontinuing the product or purchasing a sufficient volume of the obsolete part to sustain production of the product for its remaining life time (Bradley and Guerrero, (2008)). This is called a life-time or a last-time buy which is also the focus of this paper. The quantity of the final order of capital-intensive service parts should be carefully decided as the costs of obsolescence and unavailability are typically very high. It is of vital importance to balance the risk of inventory shortage versus the risk of excess inventory. On the one hand, companies are mandated to serve customers in this phase and any failure to satisfy demand for service is very costly. On the other hand, excess inventory imposes carrying cost and increases the risk of obsolescence at the end of the final phase. Many companies, such as IBM, have reported huge write-offs of inventories at the end of the service life cycle (Bulkeley, 1999).

Besides the final order, a secondary source of spare parts is to repair defective returned items. These parts may be recovered and used to service customers during the final phase. Triggered by 
a real-life business case we consider an additional spare part acquisition option, namely phaseout returns. These are returns retrieved from customers that phase out one system platform to exchange it for a new platform. Phase-out systems, however, may still be exploited to meet the demand of customers who continue to use the old platform. Due to today's replacement rates, supply of phase-outs can abound and an OEM often faces multiple phase-out occurrences in the final phase. These returns are still often in good (repairable) condition and can be used to avoid cost and to improve system performance. Examples of phase-out returns are found in various industries including aviation technologies, medical devices and military equipments.

This study is motivated by a global player in industrial automation that produces and maintains distributed plant control systems (see Krikke and van der Laan, 2011 for a detailed account). Plants are highly dependent on these control systems and therefore require prompt service when a system failure occurs. In this case, a service engineer is dispatched to the failure location immediately after the call from the customer. A spare part is taken out of the serviceable inventory to replace the failed part. The defective part is sent to a repair shop for repair. If the part is identified as repairable it may be restored with some repair effort and replenished to serviceable inventory. Recently, with the introduction of new technology, customers have been switching from existing mainframe systems to desktop based plant control systems. Therefore, planning for phase-out returns has become a challenging procedure for the OEM. The timing of system phase-outs is planned well in advance. When a customer replaces her mainframe system, the phase-out return may still serve as a source of spare parts for other customers still using the mainframe platform.

Service contracts typically run for three to five years. These predetermined service agreements oblige the company to provide its customers with a certain service level. Therefore, in case of stock-out, the company must acquire the part from a third party, thus incurring an extra cost. However, the main challenge arises when the final production quantity of a certain part/system is decided, since there are multiple sources of uncertainty including demand arrival, phase-out returns and arrival time of repairable items. The primary task is to set the final production quantity and repair policy so that it considers the possibility of phase-out returns and balances the risk of 
obsolescence with the risk of serviceable inventory stock-out.

Another reason for planning complications is the effect of phase-out returns on speculated future demand rates. Since each phase-out arrival reduces the number of installed bases in the market we expect to observe less demand for service in the future. To take this issue into account, we assume a non-stationary demand arrival process in our proposed model. Furthermore, the company is keen to glean an insight on when is the best timing to trigger a repair operation. The repair process is costly and repairing an item early causes excessive carrying cost whereas delaying repair increases the risk of shortage. Moreover, repaired and unused service parts are subject to the risk of obsolescence at the end of the final phase.

In summary, this paper addresses the inventory planning challenges of a service part in its final phase, when serviceable parts can also be acquired from the repair of failed parts and the cannibalization of system phase-outs. This particular problem was first introduced and modeled by Krikke and van der Laan (2010), but they only considered heuristic policies and heuristic optimization rules, evaluated through simulation. We take a fully analytical approach in investigating the following research questions.

- What are the characteristics of an optimal repair control policy in the final phase and how are these influenced by phase-out returns?

- What is the impact of uncertainty in the timing and quantity of phase-outs on the performance of the system? In other words, how valuable is phase-out information?

- How do heuristic repair policies perform compared to the optimal policy?

We contribute to the literature in several ways. First, we characterize the structure of the optimal policy in the final phase of the service life cycle considering phase-out occurrence. Secondly, we show that repair operations should be controlled according to a time-varying threshold level by which the system decides to trigger a repair operation based on the time remaining to the end of the horizon and the level of serviceable and repairable inventory. Thirdly, we investigate the value of phase-out information by considering cases in which phase-out schedule and quantities are subject 
to randomness and show that phase-out uncertainty should be taken into account when negotiating service agreements. Fourthly, we show that there is a considerable gap between the optimal policy and the heuristic repair control policies that have been previously suggested in the literature.

The remainder of the paper is organized as follows. Section 2 reviews the related literature. Section 3 describes the problem in detail and in section 4, we formulate the problem and characterize the structure of the optimal policy. Section 5 presents the numerical analysis and section 6 extends the primary formulation to other problems of interest considering phase-out associated uncertainties. Our conclusion and discussion can be found in section 7.

\section{Literature Review}

In this paper, we consider the inventory control of service parts of a capital goods manufacturer in the final phase of their service life cycle. The primary trade-off in this phase is balancing the risk of obsolescence and the risk of unmet service obligations. To do so, one of the main tactics used in practice is placing a final order. This problem is called final buy problem (FBP), or the end of production problem (EOP). There are three streams of research on the final phase inventory problem differentiated by the approach taken, namely service-driven, cost-driven and forecasting based approaches.

In a service-driven approach, a service level should be optimized regardless of the cost incurred by the system. Fortuin $(1980,1981)$ describes a service level approach and addresses nonrepairable items or consumable spare parts. He derives a number of curves by which the optimal final order quantity for a given service level can be obtained. Another service-driven approach is developed by van Kooten and Tan (2009) for a system in which parts are subject to the risk of condemnation. They build a transient Markovian model by which the corresponding optimal final order quantity can be obtained for a given service level.

Basically, a cost-driven approach decides on the quantity purchased by weighing the cost of ordering too many against the cost of buying too few or in other words a news-vendor problem ap- 
proach. This category includes studies by Teunter and Fortuin (1999), Teunter and Klein Haneveld (2002), Cattani and Souza (2003), Bradley and Guerrero (2009), Krikke and van der Laan (2010) and Pourakbar et. al. (2010). The latter also provides a general review on this subject.

Forecasting based approaches focus on forecasting demand for a discontinued product instead of dealing with the production or inventory problem. Moore (1971) was the first one to propose this approach followed by Ritchie and Wilcox (1977). Hong et. al. (2008) develops a stochastic forecasting model using the installed-base information to forecast the final order quantity.

This studies all focus on spare parts planning. However, there are similar problems in the context of new product introduction that deal with placing a final order for products rather than for parts. In other words, this category of works deals with the production and inventory planning when a product is replaced by its next generation counterpart. The main issues are related to the inventory planning of old and new generations of the product together with the timing of the release of the new product. We refer readers to Li et. al. (2010) and $\mathrm{Xu}$ et. al. (2010) and references therein for an overview of this stream of literature.

What distinguishes our work from the rest of the literature is that we incorporate phase-out returns as an extra source to acquire serviceable items. We discuss the complications results from phase-out returns. Moreover, we characterize the structure of the optimal policy in the final phase and show that the optimal repair policy is a time-varying decision. Numerical analysis shows the advantage of the optimal policy compared to previously developed heuristic policies, namely push and pull repair policies. Furthermore, we show that phase-out information is very valuable and significantly reduces costs.

\section{Problem Framework}

We explore the joint optimization problem of the repair control policy and final order quantity when phase-out returns occur. First, as the basic case, we consider a situation where we have perfect advance information about the schedule and the quantities of phase-outs. According to this case, $n$ 
arrivals of phase-out returns occur at times $\tau_{1}, \tau_{2}, \ldots, \tau_{n}$ in quantities equal to $o_{1}, o_{2}, \ldots, o_{n}$. Later in section 6 we show that the results can easily be extended to cases where schedule or quantities are uncertain. The problem is analyzed for a finite horizon spanning time 0 to $H$. Time 0 is defined as the beginning of the final phase and represents the point in time at which the order for the final quantity should be placed. Procuring service parts is assumed to cost $c_{p}$ per item. $H$ signifies the end of the horizon and represents the time that the last service contract expires.

Demands arrive according to a Poisson process. Each phase-out return shrinks the market size of available installed-base size. Consequently, we expect a decrease in the future demand rate for service after each phase-out arrival. Therefore, the demand arrival for service parts is modeled as a non-homogenous Poisson process. The mean value function of this process is denoted by $\Lambda(t)$. After each phase-out arrival, the demand rate drops to a lower level but stays homogenous until the next phase-out occurs. Each demand for service is coupled with a return of a defective part. With probability $q$ the returned item is repairable. Repair time is an exponential random variable and it costs $c_{r}$ per item. Uncertainty in the repair time originates from the fact that repairable returned items are received in different quality and conditions. This assumption is common in modeling remanufacturing system. It is reasonable in systems with high service time variability where repair times are typically short but occasionally longer repair times occur too. Moreover, using the memoryless property of exponential distribution gives us the opportunity to model the system as a finite horizon Markov decision process (MDP) that facilitates the analysis and makes it tractable. In case of a stock-out, the system endures a lost sale cost $c_{l}$. The demand is assumed to be lost since the service provider needs to acquire the service part through a third party.

The holding cost rates are $h_{s}$ and $h_{r}$ respectively for each unit of serviceable and repairable item per time. Any stock left at the end of the horizon is considered redundant and thus the system should dispose of it. Many countries heavily tax the disposal of parts or products. Therefore, a disposal cost of $c_{d}^{r}$ is applied for repairable items and $c_{d}^{s}$ for serviceable items such that $c_{d}^{s} \geq c_{d}^{r}$. All costs are discounted back to the beginning of the final phase with a rate $\alpha$. We note that in the formulation the cost parameters are set such that $c_{l} \geq \alpha c_{d}^{s}$. This basically means the lost sales cost 
is larger than the disposal. Thus, it is always optimal to satisfy demand when there are serviceable items.

Without loss of generality, the time $[0, H]$ is divided into time units so that the probability of having more than one demand arrival is negligible. These periods may correspond to a month, a week, a day or an hour in which both demand and phase-out arrivals happen at the beginning of each period. $o_{t}$ defined below denotes the phase-out quantity at each period

$$
o_{t}=\left\{\begin{array}{lr}
o_{i} & t=\tau_{i}, i=1,2, \ldots, n \\
0 & \text { otherwise }
\end{array}\right.
$$

Moreover, if an item is sent to the repair facility, the operation finishes in the same period with probability $\mu$. In each period, the system has to decide whether to repair an item or not. Furthermore, at the beginning of the horizon, time 0 , the system should decide how many items to order as the final order quantity.

\section{Problem Formulation}

We start with the case in which the schedule and quantities of phase-out returns are known in advance. Our objective is to find the starting inventory level at time 0 , indicating the final order quantity, and the repair control policy during the period $[0, H]$. The state of the system at an arbitrary time $t$ is defined by $(t, x, y)$, where $x$ and $y$ correspond to levels of serviceable and repairable inventories, respectively. The optimality equation at time $t$ is defined by $\nu(t, x, y)$, representing the minimum discounted cost from $t$ to $H$ with inventories $x$ and $y$ at time $t$. For $t \in[0, T]$ we have

$$
\nu(t, x, y)= \begin{cases}\nu_{11}(t, x, y) & x \geq 1, y \geq 1 \\ \nu_{01}(t, x, y) & x=0, y \geq 1 \\ \nu_{00}(t, x, y) & x=0, y=0 \\ \nu_{10}(t, x, y) & x \geq 1, y=0\end{cases}
$$


In the rest of this section we define $\nu_{11}(t, x, y), \nu_{01}(t, x, y), \nu_{00}(t, x, y)$ and $\nu_{10}(t, x, y)$. First, for the ease of representation we define $\lambda(t)$ as the demand arrival probability in period $t$. Moreover, the operator $T \nu(t+1, x, y)$ is associated with the decision of whether or not to repair an item. This operator is defined as:

$T \nu(t+1, x, y)=\min \left\{\alpha \nu(t+1, x, y), \mu\left(\alpha \nu(t+1, x+1, y-1)+c_{r}+h_{s}-h_{r}\right)+(1-\mu) \alpha \nu(t+1, x, y)\right\}$

Therefore we have:

$$
\begin{aligned}
\nu_{11}(t, x, y)= & (1-\lambda(t))\left\{h_{s} x+h_{r} y+T \nu\left(t+1, x+o_{t+1}, y\right)\right\} \\
& +(1-q) \lambda(t)\left\{h_{s}(x-1)+h_{r} y+T \nu\left(t+1, x+o_{t+1}-1, y\right)\right\} \\
& +q \lambda(t)\left\{h_{s}(x-1)+h_{r}(y+1)+T \nu\left(t+1, x+o_{t+1}-1, y+1\right)\right\}
\end{aligned}
$$

The first term on the right hand side of equation (3) represents a situation in which no demand is realized in period $t$. Therefore, the system has to decide whether to retain the state of the system or repair one item. The second term considers a situation where a non-repairable item arrives. In this case, the serviceable inventory level decreases by one unit and the system should make a decision over retaining the state of the system or repairing an item. Using the third term, the system decides on the optimal action when a repairable item arrives. In this case, the serviceable inventory decreases by one unit and the repairable inventory increases by one unit. When $x=0$ and $y \geq 1$, we have

$$
\begin{aligned}
\nu_{01}(t, x, y)= & (1-\lambda(t))\left\{h_{r} y+T \nu\left(t+1, o_{t+1}, y\right)\right\} \\
& +(1-q) \lambda(t)\left\{h_{r} y+c_{l}+T \nu\left(t+1, o_{t+1}, y\right)\right\} \\
& +q \lambda(t)\left\{h_{r} y+c_{l}+T \nu\left(t+1, o_{t+1}, y+1\right)\right\}
\end{aligned}
$$


When $x=0$ and $y=0$, in case of no demand arrival or return of a non-repairable item, the system has no option but to keep the current state of the system unchanged. However, when the returned item is repairable, the system should decide whether to send the item immediately to the repair shop or keep the current state intact. Thus, the value function in this case is defined as

$$
\begin{aligned}
\nu_{00}(t, x, y)= & (1-\lambda(t))\left\{\alpha \nu\left(t+1, o_{t+1}, 0\right)\right\} \\
& +\left\{(1-q) \lambda(t)\left\{c_{l}+\alpha \nu\left(t+1, o_{t+1}, 0\right)\right\}\right. \\
& \left.+q \lambda(t)\left\{h_{r}+c_{l}+T \nu\left(t+1, o_{t+1}, 1\right)\right\}\right\}
\end{aligned}
$$

when $x \geq 1$ and $y=0$ we have:

$$
\begin{aligned}
\nu_{10}(t, x, y)= & (1-\lambda(t))\left\{h_{s} x+\alpha \nu\left(t+1, x+o_{t+1}, 0\right)\right\} \\
& +(1-q) \lambda(t)\left\{h_{s}(x-1)+\alpha \nu\left(t+1, x+o_{t+1}-1,0\right)\right\} \\
& +q \lambda(t)\left\{h_{s}(x-1)+T \nu\left(t+1, x+o_{t+1}-1,1\right)\right.
\end{aligned}
$$

Since all leftover stocks are disposed of at the end of the horizon, time $H$, the terminal value is expressed by

$$
\nu(H, x, y)=c_{d}^{s} x+c_{d}^{r} y
$$

\subsection{Structure of the Optimal Policy}

In this section, we characterize the structure of an optimal policy. To do so, we first show that the optimal value function $\nu(t, x, y)$ satisfies certain properties enlisted in the following lemma at a specific time $t$. Next, we demonstrate that this set of properties results in a specific rule for optimal repair action which should be taken in each state. The following lemma is very helpful in characterizing the optimal policy.

Lemma 1 If $v$ is defined by equation (1) on $\left[\tau_{i}, \tau_{i+1}\right) \times \mathbb{Z}^{+} \times \mathbb{Z}^{+}$, then it satisfies the following 
properties

i) $\nu(t, x+1, y)-\nu(t, x, y)$ is non-decreasing in $x$

ii) $\nu(t, x, y+1)-\nu(t, x, y)$ is non-decreasing in $y$

Proof. See appendix.

Property $i$ ) implies that the marginal cost difference due to increasing the serviceable inventory (for a fixed level of repairable inventory) is non-decreasing. Similarly, ii) implies that the marginal cost difference due to increasing the repairable inventory (for a fixed level of serviceable inventory) is non-decreasing. In other words, $i$ and $i i$ indicate that the optimal cost function is componentwise convex in $x$ and $y$. In order to describe the optimal policy implied by the above properties, we define the following time-varying and state dependent repair threshold, $r^{*}(t, y)$ as:

$$
r^{*}(t, y)=\min \left\{x \mid \nu(t, x+1, y-1)+c_{r}-\nu(t, x, y) \geq 0, y \geq 1\right\}
$$

The simple interpretation of the above threshold level is that the system triggers a repair operation as soon as the cost of repair of one unit together with the future cost of the system becomes smaller than retaining the current state of the system. Using lemma one and the definition of the repair threshold level, the main results are stated by the following theorem.

Theorem 2 There exists an optimal time and state dependent policy that can be determined in terms of state and time dependent threshold level $r^{*}(t, y)$ as follows

I. repair an item to increase on-hand serviceable inventory if $x<r^{*}(t, y)$ and do not repair otherwise

II. $r^{*}(t, y)$ is non-increasing in time, $t \in\left[\tau_{i}, \tau_{i+1}\right)$.

Proof. See appendix.

Figure 1 illustrates an example of the optimal repair policy structure for a case without phaseout returns. As we can see, the optimal policy defines two regions in the state space. The contour of 


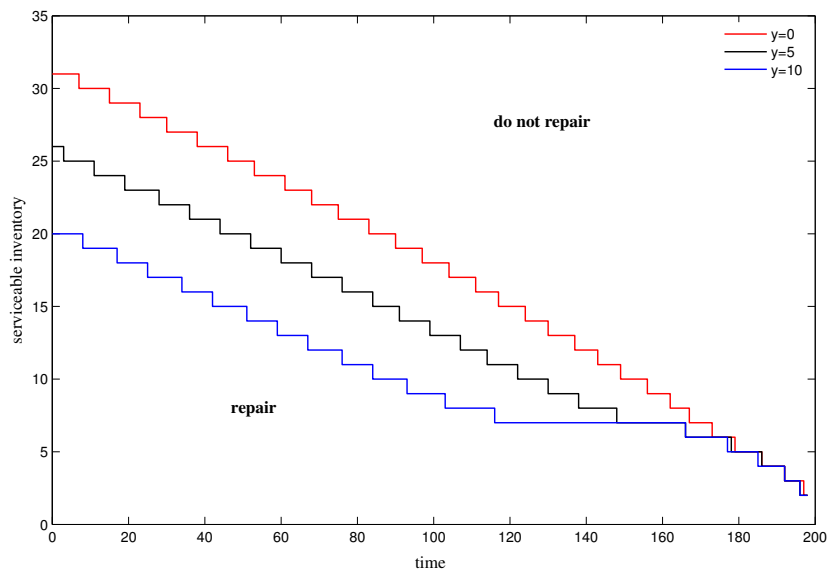

Figure 1: Optimal time and state dependent repair threshold without phase-out occurrence

this region is determined by the threshold function $r^{*}(t, y)$. The area under the contour represents the subset of the state space where the optimal decision is to repair and the area above the contour shows the portion of state space where the optimal decision is to retain the state of the system.

Figure 2 illustrates the optimal repair threshold for a case with three phase-out occurrences scheduled to arrive at times 30, 85 and 145 with quantities 7, 4 and 9 respectively. We observe that when it is closer to the end of the horizon the system prefers to have less serviceable inventory and therefore the optimal threshold shows a decreasing pattern. Moreover, having more repairable items in stock results in a smaller repair threshold. In other words, the repair threshold seems to be non-decreasing in $y$. Another observation is that the motivation for repair becomes weaker as the system approaches the next phase-out arrival and thus a lower repair threshold is set. Intuitively, the system sets a lower threshold since it is expected to receive serviceable inventory from phase-out returns.

Figures 3 and 4 show that the repair threshold is monotonic with regard to parameters $h_{s}$ and $c_{l}$. It is observed that when $h_{s}$ increases, the system tends to keep less serviceable inventory in stock. Similarly, when the stock-out penalty $c_{l}$ increases the system avoids incurring lost sale cost by increasing the repair threshold level that consequently triggers repair for higher levels of $x$.

The provisioning cost is considered a sunk cost when making decisions about repair operations. However it needs to be incorporated in the model as we have to decide upon the optimal final order 


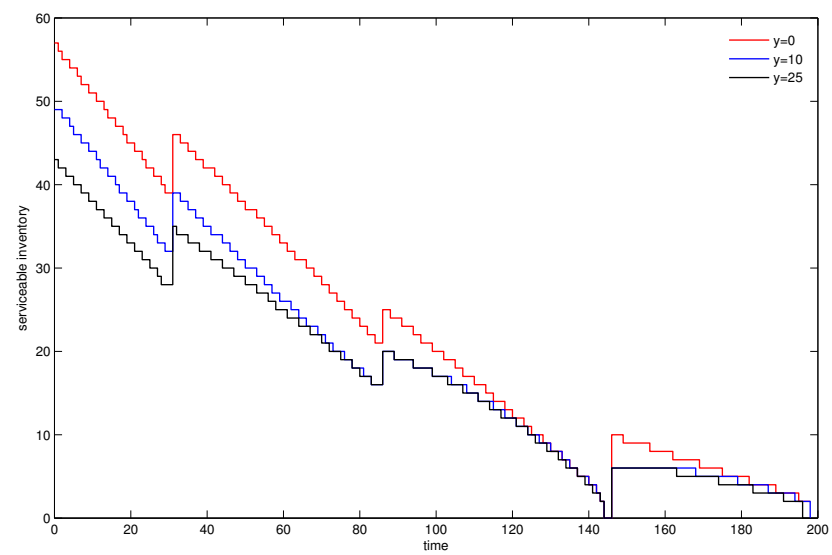

Figure 2: Optimal time and state dependent repair threshold with phase-out occurrence

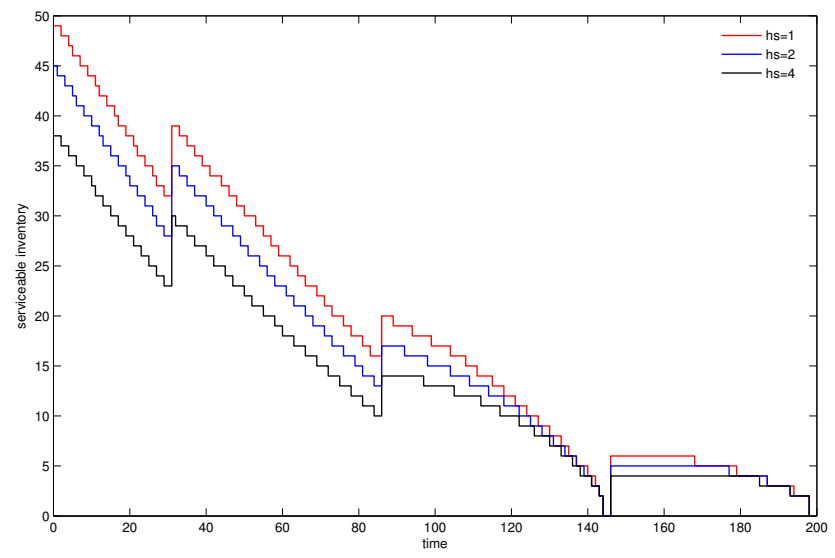

Figure 3: Effect of serviceable inventory holding cost rate on repair threshold

quantity. As an immediate result of the convexity of $\nu(t, x, y)$ with respect to $x$ the following proposition holds. Proposition 3 allows us to find the optimal final order quantity by using a simple search algorithm.

Proposition 3 The optimal order quantity is the $x$ that minimizes the following expression

$$
\nu_{0}(0, x, y)=\mathbb{E} \nu(0, x, y)+c_{p} x
$$

Proof. It is a direct result of the convexity of $\nu_{0}(0, x, y)$ in $x$. 


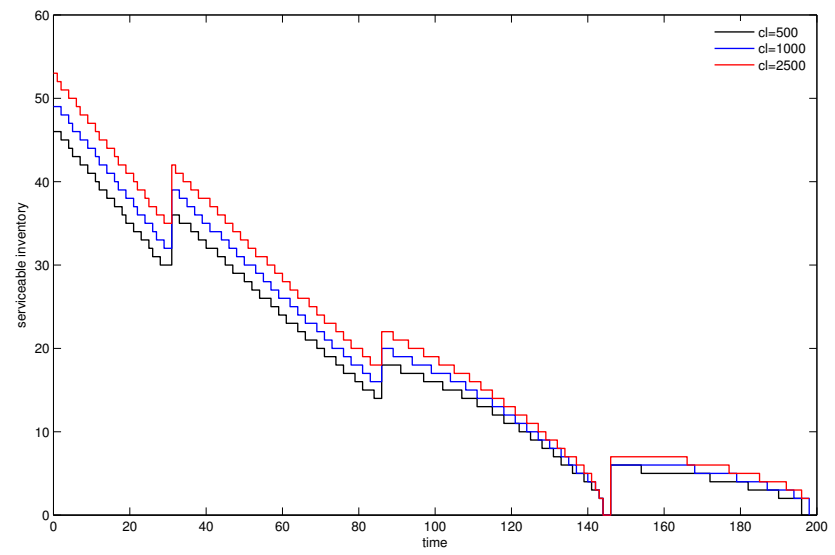

Figure 4: Effect of lost sale cost on threshold levels

The system manager is interested in the effect of the phase-out schedule on the expected total cost. Intuitively, it is not clear how postponing or pushing forward phase-out returns affects the expected total cost. Thus, we carry out an analysis in which the quantity of the first phase-out return is fixed, but the arrival schedule is varied over time. As can be observed in figures 5 and 6 , there is an optimal time to receive phase-out items. The corresponding diagram shows the effect of the phase-out schedule on the expected total cost for different quantities of the phase-out returns. When the phase-out quantity increases, the system first prefers to postpone the arrival of phase-out returns. However, when a large quantity of phase-out returns are planned to arrive the optimal schedule will push forward to the beginning of the horizon (figure 6). Furthermore, as we expect intuitively increasing the phase-out quantity results in smaller final order quantities. These effects are better represented in figure 7. As we observe for different values of $c_{l}$, when the phase-out quantities are very low or very high, the system prefers to receive them very early in time.

Therefore, when planning the inventory in the final phase, it is beneficial if the system manager can plan the schedule of phase-out arrivals and force the customers to return phase-out returns in the vicinity of the optimal schedule. 

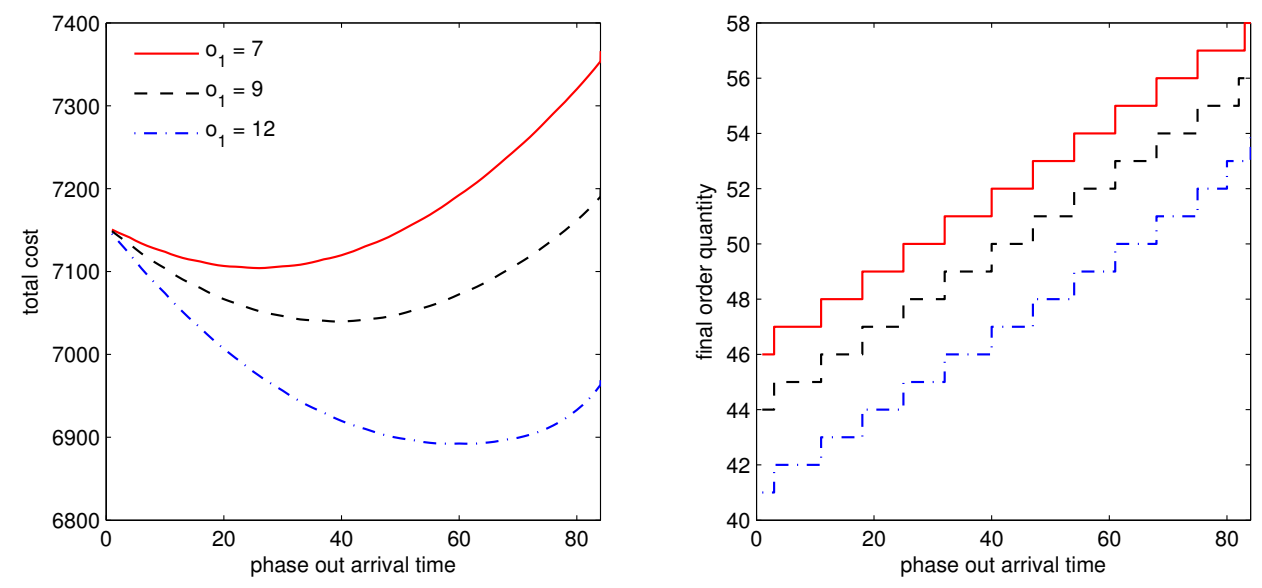

Figure 5: Effect of phase-out schedule on expected total cost
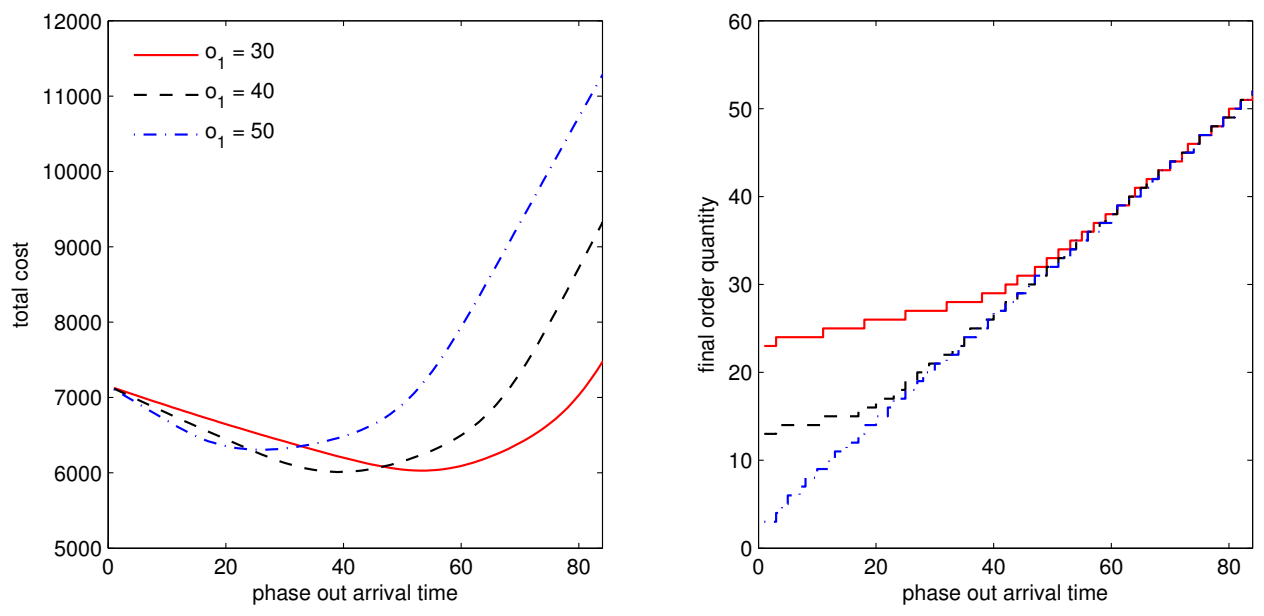

Figure 6: Effect of phase-out schedule on expected total cost 

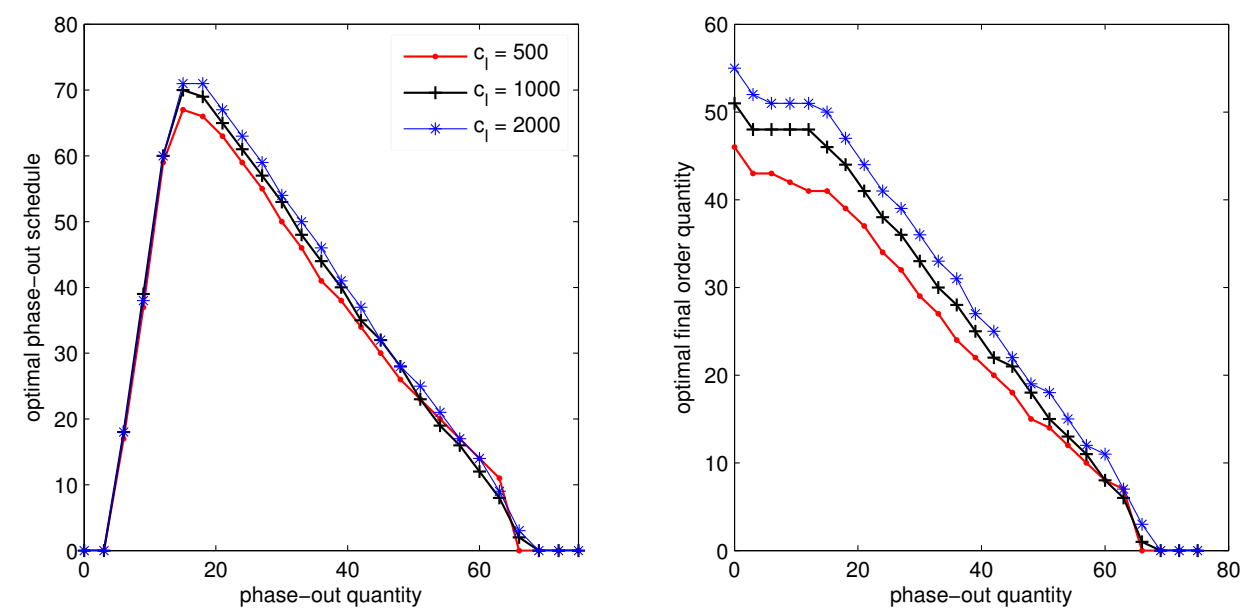

Figure 7: Effect of phase-out quantity on optimal schedule and final order quantity

\section{Numerical Analysis}

In this section, we compare the performance of some heuristic policies already developed in the literature for hybrid systems to the performance of the optimal policy for the general problem described in section 4. Our aim is to assess the value of implementing the optimal policy instead of simpler heuristics. We focus on heuristics that involve fixed (non-state dependent) parameters, since they are simpler to communicate and implement and, perhaps, are more common in practice. Below we provide a description of each of the heuristics we consider.

\subsection{Heuristic 1: Push Policy}

Under heuristic 1 , in each period $t$, if there is repairable item the system triggers repair or in other words the repairable items are immediately sent to repair shop as soon as they arrive. In this case 
the optimality equation where $x \geq 1$ and $y \geq 1$ can be expressed as

$$
\begin{aligned}
\nu_{11}(t, x, y)= & (1-\lambda(t)) \mu\left\{h_{s}(x+1)+h_{r}(y-1)+c_{r}+\alpha \nu\left(t+1, x+o_{t+1}+1, y-1\right)\right\} \\
& +(1-q) \lambda(t) \mu\left\{h_{s} x+h_{r}(y+1)+c_{r}+\alpha \nu\left(t+1, x+o_{t+1}, y-1\right)\right\} \\
& +q \lambda(t) \mu\left\{h_{s} x+h_{r} y+c_{r}+\alpha \nu\left(t+1, x+o_{t+1}, y\right)\right\} \\
& +(1-\lambda(t))(1-\mu)\left\{h_{s} x+h_{r} y+\alpha \nu\left(t+1, x+o_{t+1}, y\right)\right\} \\
& +(1-q) \lambda(t)(1-\mu)\left\{h_{s}(x-1)+h_{r} y+\alpha \nu\left(t+1, x+o_{t+1}-1, y\right)\right\} \\
& +q \lambda(t)(1-\mu)\left\{h_{s}(x-1)+h_{r}(y+1)+\alpha \nu\left(t+1, x+o_{t+1}-1, y+1\right)\right\}
\end{aligned}
$$

Relations 4-6 need to be modified based on this policy similar to (10).

\subsection{Heuristic 2: Pull Policy with a Fixed Repair Threshold}

In contrast to push, under heuristic 2 the system just repairs an item if the level of serviceable inventory is below a ceratin threshold $S$. Relation (3) according to this policy is rewritten as follows:

$$
\nu_{11}(t, x, y)=\left\{\begin{array}{rr}
\nu_{p}(t, x, y) & 1 \leq x \leq S, y \geq 1 \\
\nu_{l}(t, x, y) & x>S, y \geq 1
\end{array}\right.
$$

If $1 \leq x \leq S$, the system operates according to a push policy. Therefore, $\nu_{p}(t, x, y)$ can be

calculated according to a relation similar to relation (10). Moreover, where $x>S, y \geq 1$ the system does not trigger any repair operations and thus we have

$$
\begin{aligned}
\nu_{l}(t, x, y)= & (1-\lambda(t))\left\{h_{s} x+h_{r} y+\alpha \nu\left(t+1, x+o_{t+1}, y\right)\right\} \\
& +(1-q) \lambda(t)\left\{h_{s}(x-1)+h_{r} y+\alpha \nu\left(t+1, x+o_{t+1}-1, y\right)\right\} \\
& +q \lambda(t)\left\{h_{s}(x-1)+h_{r}(y+1)+\alpha \nu\left(t+1, x+o_{t+1}-1, y+1\right)\right\}
\end{aligned}
$$




\subsection{Experimental Design}

In our numerical test, the planning horizon is 200 periods, i.e. $H=200$. We assume that there are three planned phase-outs arriving at times 30,85 and 145 with a size of 7, 4 and 9 respectively. The demand arrival rates are set at $0.9,0.7,0.4$ and 0.2 respectively between each two consecutive phase-out returns. The other system parameters are chosen from the following sets, which generate a total of 64 different combinations. Sets are as follows $\mu \in\{0.3,0.8\}, q_{0} \in\{0.1,0.7\}, c_{l} \in$ $\{1000,2500\}, c_{r} \in\{25,125\}, c_{s s} \in\{40,120\}, h_{s} \in\{0.5,1.5\}, h_{r}=0.5$ and $c_{s r}=40$.

We compute the cost and final order quantity for each instance with different parameters under optimal and heuristic policies. We report the average, minimum and maximum cost increase resulting from each heuristic policy compared to the optimal policy. We define the cost increase by the following expression

$$
\Delta_{\text {cost }}=\frac{T C_{h}-T C_{o p t}}{T C_{o p t}} \times 100 \%
$$

where, $T C_{h}$ and $T C_{\text {opt }}$ are expected cost according to heuristic and optimal policies, respectively.

The numerical results in table 1 show that on average the pull policy outperforms the push policy, as intuition might dictate. This is because while repair is triggered according to a push policy, the system does not utilize any information regarding time and state of the system. However, the pull policy benefits from deliberately postponing the repair operation. The average cost increase for a pull policy is $6.83 \%$ compared to $20.75 \%$ for a push policy. Moreover, in some cases implementing a push or pull policy instead of an optimal policy can impair the performance of the system as much as $43.68 \%$ or $31.10 \%$, respectively. Moreover, on average the pull policy tends to place a larger final order quantity than the optimal policy, whereas push always places a smaller final order quantity. This can be easily justified considering the structure of each policy. Basically, the system expects to yield more serviceable items through repair when it operates according to a push rather than a pull policy. Therefore, the final order quantity is smaller when the repair operation is controlled according to a push structure. 
Table 1: The Value of the Optimal Policy

\begin{tabular}{|c|c|c|c|}
\hline & $\Delta_{\text {cost }} \%$ & Max $\Delta_{\text {cost }} \%$ & Min $\Delta_{\text {cost }} \%$ \\
\hline Push & 20.75 & 43.68 & 3.70 \\
\hline Pull & 6.83 & 31.10 & 0.35 \\
\hline
\end{tabular}

Table 2: Final order quantity

\begin{tabular}{|c|c|c|c|}
\hline & Average $n$ & Max $n$ & Min $n$ \\
\hline Optimal & 61.76 & 83 & 39 \\
\hline Push & 59.78 & 82 & 37 \\
\hline Pull & 64.75 & 85 & 42 \\
\hline
\end{tabular}

\subsection{Impact of Cost Terms}

In this section, we first aim at exploring the impact of various cost parameters on the value of the optimal control policy and secondly at understanding better the performance of push and pull policies. To achieve this, we assign different values to a specific parameter and keep other variables constant. For the base case scenario the parameters are set as shown in table 1. Figure 8 suggests that there are many situations for which a push might outperform a pull policy. Figure 8.a implies that as the holding cost for repairable items increases, the average error of a push policy decreases whereas it increases for a pull policy. This is because it becomes more costly to hold repairable items which makes push more appealing. Therefore, for larger values of $h_{r}$ push becomes superior to pull. Moreover, as the serviceable item holding cost rate increases push becomes less attractive and therefore pull outperforms push for larger $h_{s}$, as shown in figure 8.b.

Next, we examine the impact of the repair rate on the value of the optimal policy (figure 8.c). As the repair rate increases, pull tends to outperform push policy. This is because when the repair rate is high, the repair operation becomes more reliable and therefore the system prefers to postpone the

Table 3: Base case parameters

\begin{tabular}{|c|c|c|c|c|c|c|c|c|}
\hline$c_{p}$ & $c_{l}$ & $c_{r}$ & $h_{r}$ & $h_{n}$ & $c_{d}^{s}$ & $c_{d}^{r}$ & $q$ & $\mu$ \\
\hline 200 & 1000 & 75 & 0.5 & 1 & 80 & 40 & 0.3 & 0.4 \\
\hline
\end{tabular}


a.

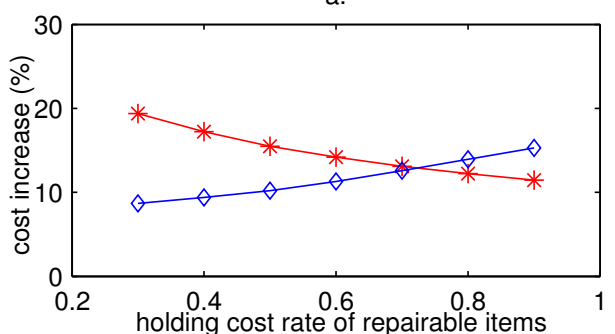

c.
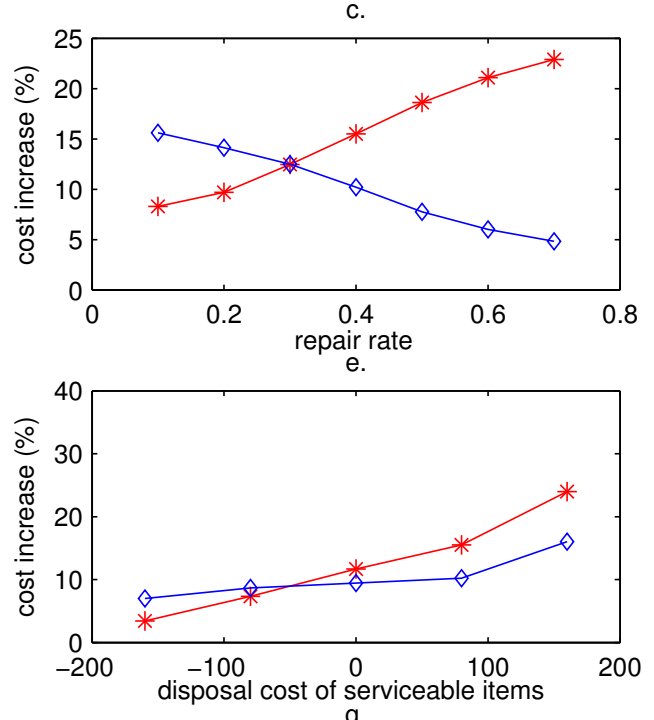

g.

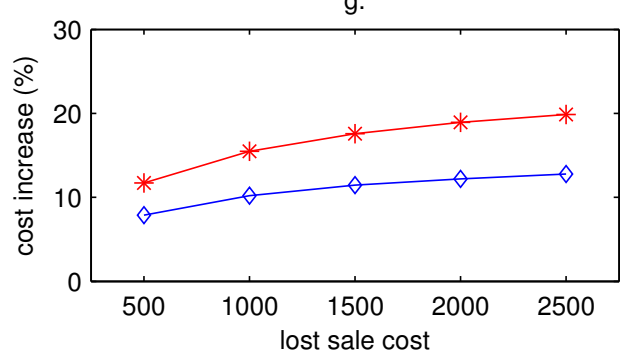

b.
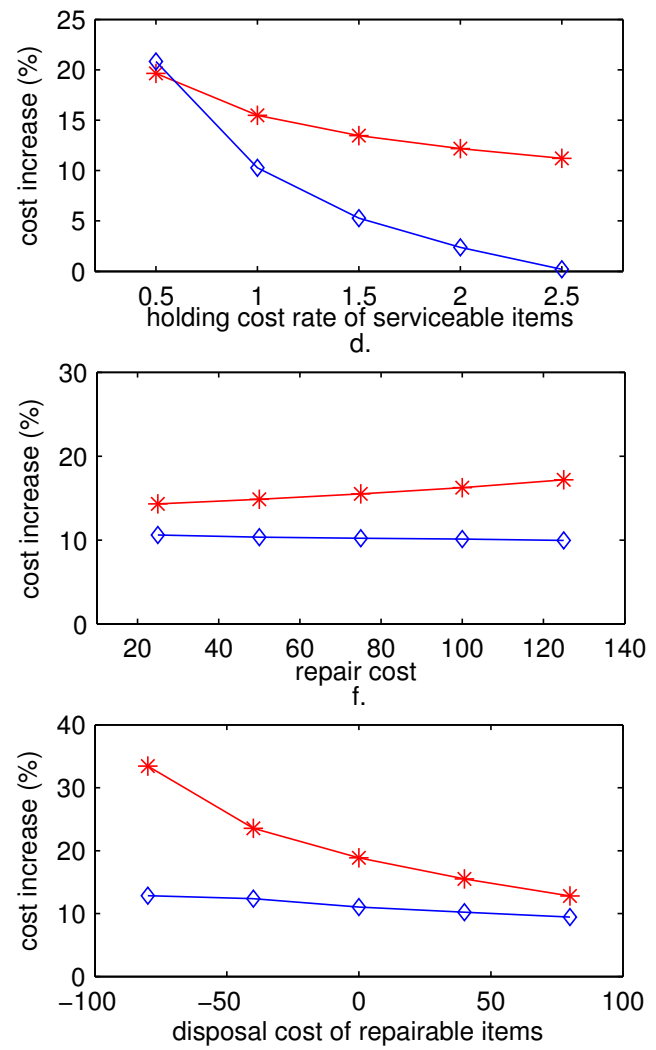

* Push Policy

Figure 8: Impact of various parameters on the performance of heuristic policies 
repair operation and trigger it when the serviceable inventory hits a certain level. Since repairable items are cheaper to hold, the system can benefit more from pull than push when repair can be completed more swiftly. Furthermore, the increase in repair cost seems to impair the performance of the push more than pull policy (figure 8.d). This is mainly due to the fact that pull can adjust $S$ in order to avoid extra repair cost.

As intuition dictates, push policy outperforms pull when the disposal of the serviceable item generates a large revenue (figure 8.e). As the serviceable item disposal cost increases, push loses

its advantage and therefore pull tends to be a more effective policy. When it becomes more costly to dispose of repairable items, then having less repairable stock at the end of the horizon becomes more advantageous. Hence, we observe that the performance of the push policy improves when $c_{d}^{r}$ increases (figure 8.f). Figure 8.g suggests that with the increase of the lost sale cost, both push and pull policies impair performance significantly.

\section{Extension to the Other Cases}

Our model and approach can accommodate several related problems of interest as simple extensions. In this section, we describe some of these cases. The main interest lies with phase-out uncertainty. In practice, the phase-out arrival schedule is subject to considerable uncertainty. This is because in order to switch from an old platform to a new one, the customer has to take many sequential steps including purchasing, testing, personnel training etc. All of these activities are subject to randomness that might render an uncertain phase-out arrival schedule. Furthermore, in some cases, the OEM is not fully aware of the condition of platforms in use and therefore the phase-out quantity is also subject to randomness. First, we extend the model to a case with phaseout schedule uncertainty and then to a case with uncertain schedule as well as uncertain quantity. 


\subsection{Phase-out Schedule is Stochastic While Quantities are Deterministic}

In this case, the arrival of phase-out returns are subject to uncertainty, but we can limit the arrival time, $\tau_{i}$, to the interval $\left[\underline{\tau}_{i}, \bar{\tau}_{i}\right]$ with arrival probability $\mathbb{P}\left\{\tau_{i}=t\right\}=p_{i}$. In order to calculate the policy cost, we define the function $\omega\left(\tau_{i}, \tau_{i+1}, x, y\right)$ as the cost function of running the policy from time $\tau_{i}$ to $\tau_{i+1}$. Therefore, the expected total cost function from time 0 to $H$ is obtained by

$$
\mathbb{E} \omega(x, y)=\sum_{i=0}^{n} \sum_{\tau_{i}=\underline{\tau}_{i}}^{\bar{\tau}_{i}} \sum_{\tau_{i+1}=\underline{\tau}_{i+1}}^{\bar{\tau}_{i+1}} p_{i}\left(\tau_{i}\right) p_{i+1}\left(\tau_{i+1}\right) \omega\left(\tau_{i}, \tau_{i+1}, x, y\right)
$$

where $\tau_{0}=0$ and $\tau_{n+1}=T$. Moreover, $\omega\left(\tau_{i}, \tau_{i+1}, x, y\right)$ for $\tau_{i} \leq t<\tau_{i+1}$ is defined as

$$
\omega\left(\tau_{i}, t, x, y\right)= \begin{cases}\omega_{11}\left(\tau_{i}, t, x, y\right) & x \geq 1, y \geq 1 \\ \omega_{01}\left(\tau_{i}, t, x, y\right) & x=0, y \geq 1 \\ \omega_{00}\left(\tau_{i}, t, x, y\right) & x=0, y=0 \\ \omega_{10}\left(\tau_{i}, t, x, y\right) & x \geq 1, y=0\end{cases}
$$

which can be calculated in the same fashion as (3) and (4).

In order to investigate the effect of phase-out uncertainty, we limit our attention to the first phase-out arrival and use the variance in the arrival schedule as a measure for uncertainty. As intuition dictates, more variation around the optimal phase-out schedule results in a higher expected total cost. The results are shown in figure 9. It is observed that the system places a larger final order quantity in order to hedge against uncertainty when the coefficient of variation of the phaseout schedule increases.

\subsection{Both Phase-out Schedule and Quantities are Stochastic}

In this situation, the quantity of phase-out returns is also subject to uncertainty. With probability $q_{i}(k), k$ parts out of $o_{i}$ can immediately be added to the serviceable inventory and the rest should 

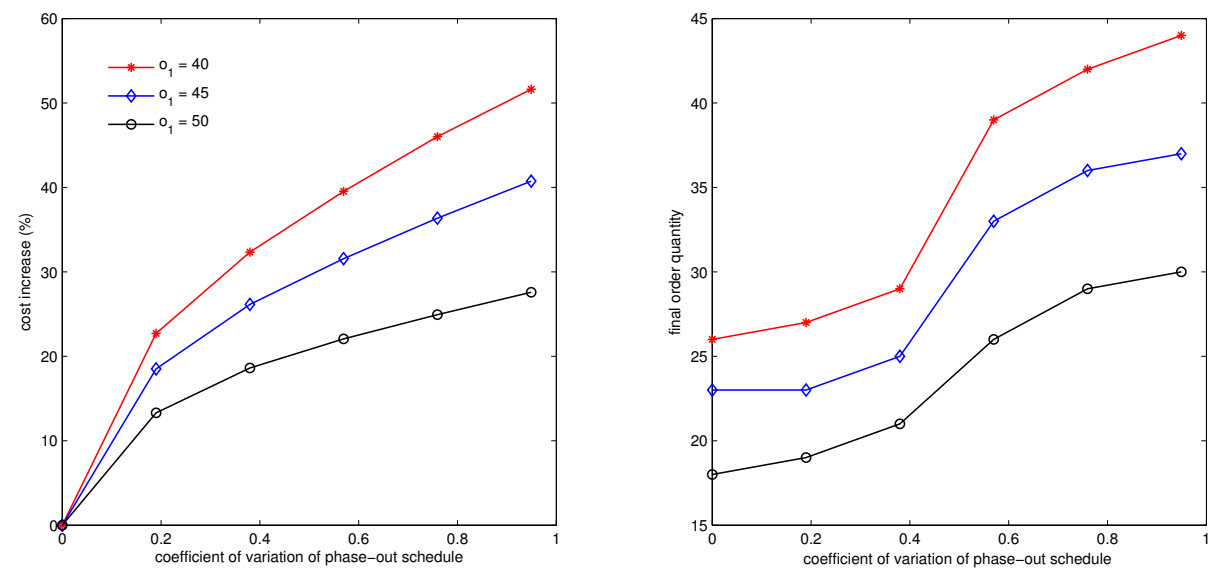

Figure 9: Effect of phase-out arrival uncertainty on expected total cost
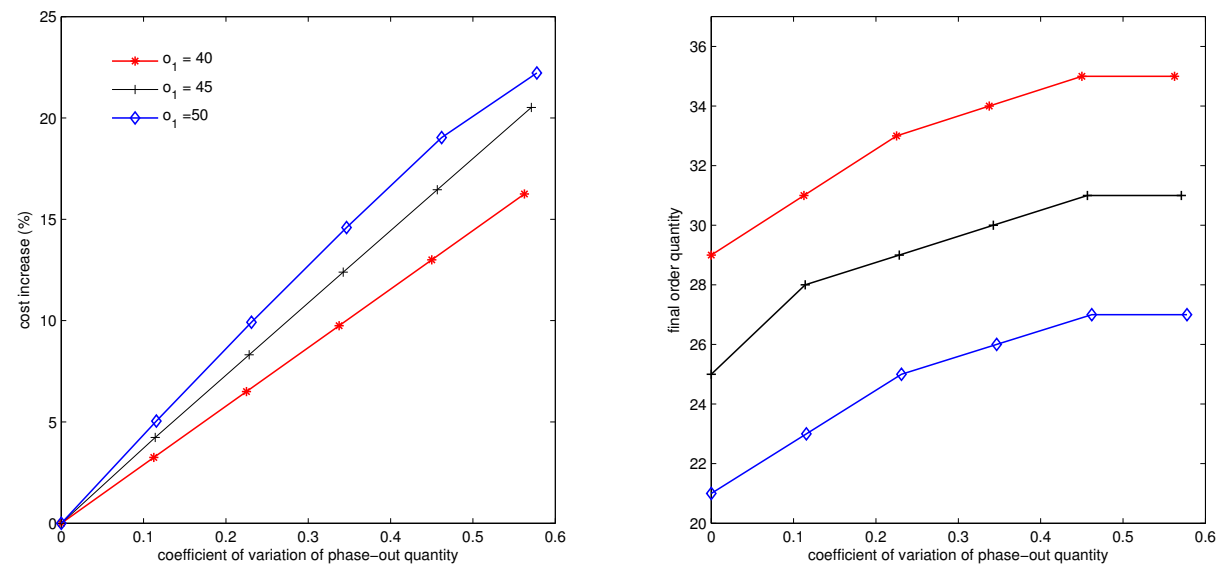

Figure 10: Effect of phase-out quantity uncertainty on expected total cost

undergo repairs. In this case, the expected total cost function from time 0 to $H$ is obtained by

$$
\mathbb{E} \omega(x, y)=\sum_{i=0}^{n} \sum_{\tau_{i}=\underline{\tau}_{i}}^{\bar{\tau}_{i}} \sum_{\tau_{i+1}=\underline{\tau}_{i+1}}^{\bar{\tau}_{i+1}} \sum_{k=0}^{o_{i}} \sum_{j=0}^{o_{i}-k} q_{i}(k) p_{i}\left(\tau_{i}\right) p_{i+1}\left(\tau_{i+1}\right) \omega\left(\tau_{i}, t+1, x+k, y+j\right)
$$

where $\omega\left(\tau_{i}, t+1, x+k, y+j\right)$ can be calculated similar to (14)

In order to investigate the effect of phase-out quantity uncertainty, we keep the standard deviation of the phase-out schedule at a fixed level and change the coefficient of variation of the phase-out quantity while its expected value is fixed. The results are shown in figure 10. As can be observed, more randomness in phase-out quantities results in an increase of final order quantity 
and consequently impairs the performance of the system.

\section{Discussion and Conclusion}

In this paper we address the end-of life inventory and repair planning for service parts in their final phase. In particular, we focus on the final order quantity, taking into account product returns due to failures and phase-outs and (optimal) repair policy.

When the schedule and quantities of phase-out returns are known in advance we proved that the structure of the optimal policy is characterized in terms of state and time dependent threshold levels $r^{*}(t, y)$, where $t$ is time and $y$ is the remanufacturable inventory. So, whenever the level of serviceable inventory $x$ is below $r^{*}(t, y)$, a part, if available, is sent to the repair shop.

Varying the arrival time of phase-outs, we see that there is an optimal timing of phase out returns for a given phase-out quantity. When phase-out quantities are higher, the optimal timing first moves later in time, but eventually moves earlier in time. Therefore, in constructing service contracts it is important to take the timing of phase-outs into account or even negotiate optimal timing.

The optimal policy combines push and pull in one policy. Simpler policies have previously been developed and are widely used in practice, such as push (push repairable items upon arrival) and pull (pull repairable items relative to a fixed serviceable inventory level). Our numerical study suggests that push only outperforms pull when the holding cost rates for serviceable and remanufacturable items are very close or when the repair rate is very small. The difference between push and pull becomes smaller as the disposal cost for a remanufacturable part increases. For our parameter setting, the optimal policy outperforms pull by up to $31.10 \%$ (6.83\% on average). When the serviceable holding cost rate or the probability that a part is remanufacturable is high, the performance of pull is close to optimal. With respect to the final order quantity decision we see that push always underestimates the optimal final order quantity, whereas pull always overestimates it.

Another finding highlight the vital importance of phase-out information. We show that un- 
certainties about the phase-out schedule and quantity can impair the performance of the system. Having accurate information over phase-out returns can lead to considerable cost savings.

Even though the final phase is known as the longest phase of the service life cycle, it has received little attention in the literature. Our work can be extended in various dimensions, for instance, to a situation in which the OEM is allowed to dispose of inventories during the course of the final phase. Another important extension is dealing with end-of-life inventory planning in the presence of differentiated customers based on service contracts or equipment criticalness.

\section{References}

[1] Bradley J.R., H.H. Guerrero. 2009. Life-Time Buy Decisions with Multiple Parts, Production and Operations Management, 18(1).

[2] Bulkeley W.M. . IBM. 1999. Had 98 PC pretax loss of nearly \$ 1 billion, The Wall Street Journal, March 25.

[3] Cattani K.D., G.C. Souza. 2003. Good buy? Delaying end-of-life purchases, European Journal of Operational Research, 146 216-228.

[4] Fortuin L. 1980. The All-Time Requirement of Spare Parts for Service After Sales- Theoretical Analysis and Practical Results, International Journal of Operations and Production Management, 1(1), 59-70.

[5] Fortuin L. 1981. Reduction of All-time requirements for Spare Parts, International Journal of Operations and Production Management, 2(1), 29-37.

[6] Hong J.S., H.Y. Koo, C.S. Lee, J. Ahn. 2008. Forecasting service parts demand for a discontinued product, IIE Transactions 40 640-649.

[7] Klein Haneveld W.K., R.H. Teunter. 1998. The Final Order Problem, European Journal of Operational Research, 107 35-44. 
[8] Kooten J.P.J. van, T. Tan. 2009. The Final Order Problem for Repairable Spare Parts under Condemnation. Journal of the Operational Research Society, 6014491461.

[9] Krikke H.R., Laan E. van der. 2011. Last Time Buy and Control Policies With Phase-Out Returns: A Case Study in Plant Control Systems, International Journal of Production Research.

[10] Li H, S.C. Graves, D.B. Rosenfield. 2010. Optimal Planning Quantities for Product Transition , Production and Operations Management, 19(2) 142-155.

[11] Moore J.R. 1971. Forecasting and Scheduling for Past-Model Replacement Parts, Management Science 18 B200-B213.

[12] Teunter R.H., L. Fortuin. 1998. End-of-life service: A case study, European Journal of Operational Research, 107 19-34.

[13] Teunter R.H., W.K. Klein Haneveld. 2002. Inventory control of service parts in the final phase, European Journal of Operational Research 137 497-511.

[14] Pourakbar M., J.B.G. Frenk, R. Dekker. 2010. End-of-life inventory decisions for consumer electronics service parts, econometric institute report series, Erasmus University Rotterdam.

[15] Ritchie E., P.Wilcox. 1977. Renewal Theory Forecasting for Stock Control, Jornal of the Operational Research Society, 1 90-93.

\section{APPENDIX}

\section{Proof of Lemma 1}

Proof. First, for the sake of brevity we define $\xi(t, x, y)=\nu_{11}(t, x, y)-\nu_{11}(t, x-1, y)$. Then, we need to show that $\xi(t, x, y)$ is non-decreasing in $x$. Moreover, we define $\xi(t, 0, y)=\alpha / c_{l}$. We follow an induction approach in order to show the desired results.

From the terminal value definition we have $\nu_{11}(H, x, y)=c_{d}^{s} x+c_{d}^{r} y$, thus, $\xi(H, x, y)=c_{d}^{s}$. From assumption (1) we have $c_{l} \geq \alpha c_{d}^{s}$ which can be immediately translated to $\xi(H, 1, y) \geq$ $\xi(H, 0, y)$. Therefore, $\xi(H, x, y)$ is non-decreasing in $x$. 
Following the induction, we assume $\xi(t-1, x, y)$ is non-decreasing in $x$, then we need to show that $\xi(t, x, y)$ is non-decreasing in $x$. From the definition of $r^{*}(t, y)$ we know that there exists a $r^{*}(t, y)$ such that if $x \geq r^{*}(t, y)$ then it is optimal not to repair and repair otherwise. It is worth nothing that $\lambda(t)=\lambda$ for $t \in\left[\tau_{i}, \tau_{i+1}\right)$. Then from equation (3) we have

$$
\begin{aligned}
& \xi(t-1, x, y)= \\
& (1-\lambda)\left\{h_{s}+\alpha \xi(t, x, y) \mathbf{1}_{\left\{x \geq r^{*}(t, y)\right\}}\right\} \\
& +(1-q) \lambda\left\{h_{s}+\alpha \xi(t, x-1, y) \mathbf{1}_{\left\{x-1 \geq r^{*}(t, y)\right\}}\right\} \\
& +q \lambda\left\{h_{s}+\alpha \xi(t, x-1, y+1) \mathbf{1}_{\left\{x-1 \geq r^{*}(t, y+1)\right\}}\right\} \\
& +(1-\lambda)\left\{h_{s}+(1-\mu) \alpha \xi(t, x, y) \mathbf{1}_{\left\{x<r^{*}(t, y)\right\}}+\mu\left(\alpha \xi(t, x+1, y-1)+c_{r}\right) \mathbf{1}_{\left\{x<r^{*}(t, y)\right\}}\right\} \\
& +(1-q) \lambda\left\{h_{s}(1-\mu) \alpha \xi(t, x-1, y) \mathbf{1}_{\left\{x-1<r^{*}(t, y)\right\}}+\mu\left(\alpha \xi(t, x, y-1)+c_{r}\right) \mathbf{1}_{\left\{x-1<r^{*}(t, y)\right\}}\right\} \\
& +q \lambda\left\{h_{s}+(1-\mu) \alpha \xi(t, x-1, y+1) \mathbf{1}_{\left\{x-1<r^{*}(t, y+1)\right\}}+\mu\left(\alpha \xi(t, x, y)+c_{r}\right) \mathbf{1}_{\left\{x-1<r^{*}(t, y)\right\}}\right\}
\end{aligned}
$$

since $\xi(t-1, x, y)$ is a linear expression of $\xi(t, x, y)$ and using induction assumption we know that $\xi(t-1, x, y)$ is non-decreasing in $x$ therefore $\xi(t-1, x, y)$ is non-decreasing in $x$. The same can be established for expressions (6), (8) and (10).

Using the same approach and the fact that $h_{s} \geq h_{r}$ we can show that $\xi(t, x, y)$ is non-decreasing in $y$.

\section{Proof of Theorem 2}

I. The results are intuitive considering the definition of the repair threshold and componentwise convexity properties.

II.

Proof. We show this by induction where the induction assumption is $r^{*}(t, y) \leq r^{*}(t-1, y)$ and $\beta(t-1, x, y) \geq \beta(t, x, y)$, where $\beta(t, x, y)=\nu_{11}(t, x+1, y-1)-\nu_{11}(t, x, y)$. It is straightforward 
to show the results for $t=H$, since intuitively $r^{*}(H, y)=0 \leq r^{*}(H-1, y)$.

Then, assuming the induction assumption holds at time $t-1$ we need to show that it holds at time $t$. In order to show that the induction assumption holds we employ a contradiction approach. Suppose for contradiction that $r^{*}(t-1, y)<r^{*}(t, y)$. Using the definition of repair threshold it immediately implies that $\beta\left(t, r^{*}(t-1, y), y\right)+c_{r} \geq 0$. Using (3), we should note that

$$
\begin{aligned}
\beta(t-1, x, y)= & (1-\lambda)\left\{\alpha \beta(t, x, y) \mathbf{1}_{\left\{x \geq r^{*}(t, y)\right\}}\right\} \\
& +(1-q) \lambda\left\{\alpha \beta(t, x-1, y) \mathbf{1}_{\left\{x-1 \geq r^{*}(t, y)\right\}}\right\} \\
& +q \lambda\left\{\alpha \beta(t, x-1, y+1) \mathbf{1}_{\left\{x-1 \geq r^{*}(t, y+1)\right\}}\right\} \\
& +(1-\lambda)\left\{(1-\mu) \alpha \beta(t, x, y) \mathbf{1}_{\left\{x<r^{*}(t, y)\right\}}+\mu \alpha \beta(t, x+1, y-1) \mathbf{1}_{\left\{x<r^{*}(t, y)\right\}}\right\} \\
& +(1-q) \lambda\left\{(1-\mu) \alpha \beta(t, x-1, y) \mathbf{1}_{\left\{x-1<r^{*}(t, y)\right\}}+\mu \alpha \beta(t, x, y-1) \mathbf{1}_{\left\{x-1<r^{*}(t, y)\right\}}\right\} \\
& +q \lambda\left\{(1-\mu) \alpha \beta(t, x-1, y+1) \mathbf{1}_{\left\{x-1<r^{*}(t, y+1)\right\}}+\mu \alpha \beta(t, x, y) \mathbf{1}_{\left\{x-1<r^{*}(t, y)\right\}}\right\}
\end{aligned}
$$

For the ease of representation we define $\Delta_{t} \beta(t, x, y)=\beta(t, x, y)-\beta(t+1, x, y)$, Then we 
have

$$
\begin{aligned}
& \Delta_{t} \beta\left(t-1, r^{*}(t, y), y\right)=(1-\lambda)\left\{\alpha \Delta_{t} \beta\left(t, r^{*}(t, y), y\right) \mathbf{1}_{\left\{r^{*}(t, y) \geq r^{*}(t+1, y)\right\}}\right\} \\
& +(1-q) \lambda\left\{\alpha \Delta_{t} \beta\left(t, r^{*}(t, y)-1, y\right) \mathbf{1}_{\left\{r^{*}(t, y)-1 \geq r^{*}(t+1, y)\right\}}\right\} \\
& +q \lambda\left\{\alpha \Delta_{t} \beta\left(t, r^{*}(t, y)-1, y+1\right) \mathbf{1}_{\left\{r^{*}(t, y+1)-1 \geq r^{*}(t+1, y+1)\right\}}\right\} \\
& +(1-\lambda)\left\{(1-\mu) \alpha \Delta_{t} \beta\left(t, r^{*}(t, y), y\right) \mathbf{1}_{\left\{r^{*}(t, y)<r^{*}(t+1, y)\right\}}\right\} \\
& +(1-\lambda) \mu \alpha \Delta_{t} \beta\left(t, r^{*}(t, y)+1, y-1\right) \mathbf{1}_{\left\{r^{*}(t, y)<r^{*}(t+1, y)\right\}} \\
& +(1-q) \lambda\left\{(1-\mu) \alpha \Delta_{t} \beta\left(t, r^{*}(t, y)-1, y\right) \mathbf{1}_{\left\{r^{*}(t, y)-1<r^{*}(t+1, y)\right\}}\right\} \\
& +(1-q) \lambda \mu \alpha \Delta_{t} \beta\left(t, r^{*}(t, y), y-1\right) \mathbf{1}_{\left\{r^{*}(t, y)-1<r^{*}(t+1, y)\right\}} \\
& +q \lambda\left\{(1-\mu) \alpha \Delta_{t} \beta\left(t, r^{*}(t, y)-1, y+1\right) \mathbf{1}_{\left\{r^{*}(t, y+1)-1<r^{*}(t+1, y+1)\right\}}\right\} \\
& +q \lambda \mu \alpha \Delta_{t} \beta\left(t, r^{*}(t, y), y\right) \mathbf{1}_{\left\{r^{*}(t, y)-1<r^{*}(t+1, y)\right\}}
\end{aligned}
$$

Using the induction assumption, we have the terms $\Delta_{t} \beta(t, x-1, y+1), \Delta_{t} \beta(t, x-1, y)$ and $\Delta_{t} \beta(t, x, y)$ are all non-negative. Therefore, $\Delta_{t} \beta(t-1, x, y)=\beta(t-1, x, y)-\beta(t, x, y) \geq 0$. Thus,

$$
\beta(t-1, x, y)+c_{r} \geq \beta(t, x, y)+c_{r} \geq 0
$$

which implies that $r^{*}(t, y) \leq r^{*}(t-1, y)$, it is a contradiction.

Next, we show $\beta(t-1, x, y) \geq \beta(t, x, y)$ given that $r^{*}(t, y) \leq r^{*}(t-1, y), t \in(0, H]$. We separate the interval $\left(0, r^{*}(t-1, y)\right]$ into the following subintervals $\left(0, r^{*}(t+1, y)\right],\left(r^{*}(t+\right.$ $\left.1, y), r^{*}(t, y)\right]$ and $\left(r^{*}(t, y), r^{*}(t-1, y)\right]$. For each of these intervals we show the results. It is worth mentioning that we use the notation $\Delta_{t} \beta(t, x, y)$ as defined previously. Moreover, we just show the results for the case that $x \geq 1$ and $y \geq 1$, a similar analysis can be applied to other cases.

Case 1. $\forall x \in\left(0, r^{*}(t+1, y)\right]$ 
In this case we have

$$
\begin{aligned}
& \Delta_{t} \beta(t-1, x, y)= \\
& (1-\lambda)\left\{(1-\mu) \Delta_{t} \beta(t, x, y) \mathbf{1}_{\left\{r^{*}(t, y)<r^{*}(t+1, y)\right\}}+\mu \Delta_{t} \beta(t, x+1, y-1) \mathbf{1}_{\left\{r^{*}(t, y)<r^{*}(t+1, y)\right\}}\right\} \\
& +(1-q) \lambda\left\{(1-\mu) \Delta_{t} \beta(t, x-1, y) \mathbf{1}_{\left\{r^{*}(t, y)-1<r^{*}(t+1, y)\right\}}+\mu \Delta_{t} \beta(t, x, y-1) \mathbf{1}_{\left\{r^{*}(t, y)-1<r^{*}(t+1, y)\right\}}\right\} \\
& +q \lambda\left\{(1-\mu) \Delta_{t} \beta(t, x-1, y+1) \mathbf{1}_{\left\{r^{*}(t, y+1)-1<r^{*}(t+1, y+1)\right\}}+\mu \Delta_{t} \beta(t, x, y) \mathbf{1}_{\left\{r^{*}(t, y)-1<r^{*}(t+1, y)\right\}}\right\}
\end{aligned}
$$

Following an approach similar to the previous case we can show that the right hand side are all non-negative and therefore $\Delta_{t} \beta(t-1, x, y) \geq 0$.

Case 2. $\forall x \in\left(r^{*}(t+1, y), r^{*}(t, y)\right]$

$$
\begin{aligned}
\Delta_{t} \beta(t-1, x, y)= & (1-\lambda)\left\{\Delta_{t} \beta(t, x, y) \mathbf{1}_{\left\{r^{*}(t, y) \geq r^{*}(t+1, y)\right\}}\right\} \\
& +(1-q) \lambda\left\{\Delta_{t} \beta(t, x-1, y) \mathbf{1}_{\left\{r^{*}(t, y)-1 \geq r^{*}(t+1, y)\right\}}\right\} \\
& +q \lambda\left\{\Delta_{t} \beta(t, x-1, y+1) \mathbf{1}_{\left\{r^{*}(t, y+1)-1 \geq r^{*}(t+1, y+1)\right\}}\right\}
\end{aligned}
$$

Following an approach similar to the previous case we can show that the right hand side are all non-negative and therefore $\Delta_{t} \beta(t-1, x, y) \geq 0$.

Case 3. $\forall x \in\left(r^{*}(t, y), r^{*}(t-1, y)\right]$

In this case we have $r^{*}(t, y)<x \leq r^{*}(t-1, y)$, then according to the definition of $r^{*}(t, y)$ we have $\beta(t-1, x, y)+c_{r} \geq 0$ and $\beta(t, x, y)+c_{r} \leq 0$ therefore $\beta(t-1, x, y)-\beta(t, x, y) \geq 0$.

These show the desired results for $x \geq 1$ and $y \geq 1$. We can follow a similar approach for other cases. 